\title{
ATENCIÓN A LAS NECESIDADES DE SALUD DE LOS HOMBRES EN LA CONSULTA DE PLANIFICACIÓN FAMILIAR

\author{
ATTENTION TO THE HEALTH NEEDS \\ OF MEN IN FAMILY PLANNING \\ CONSULTATION
}

Lic. Yoanna Martínez Boloña.

yoanna@ensap.sld.cu

Licenciada en Sociología, profesora instructora de la Ensap.

\section{Dra C. Zoe Díaz Bernal.}

zoe@ensap.sld.cu

Lic. Microbiología, máster en Antropología. Profesora titular de la Ensap.

\section{RESUMEN}

La Consulta de Planificación Familiar (CPF) es un espacio creado para orientar y facilitar el proceso de toma de decisiones que una pareja hace respecto al tipo de familia que desea conformar y al proyecto de vida familiar que planean construir. La prestación del servicio ha sido descrita en la literatura científica como poco inclusiva para los hombres, a pesar de la influencia que ellos ejercen en la salud de sus parejas sexuales y en su propia salud sexual. Determinar a través de las percepciones de los/las prestadores/ as de un servicio de planificación familiar si la atención que brindan satisface las necesidades de los hombres. La investigación consistió en un estudio de carácter descriptivo de corte transversal, realizado en el Policlínico Docente "Antonio Maceo". Se aplicaron dos técnicas: entrevista en profundidad y observación. La praxis de las prestadoras es poco inclusiva de las necesidades de atención masculina, esto no puede interpretarse como una falta de intención de esas profesionales al respecto, sino como expresión y reforzamiento de un hacer basado más en la experiencia social, con base cultural, que en los enfoques científicos y socioculturales más contemporáneos, sobre equidad de género y su determinación en la salud. El servicio brindado por el personal de salud de la Consulta de Planificación Familiar no satisface las necesidades de atención relativas a su salud sexual y reproductiva de los hombres, sino a la atención de las identificadas por y para mujeres.

Palabras clave: Planificación familiar. Hombres. Necesidades de atención.

\section{ABSTRACT}

Family Planning Consultation (CPF) is a space created to guide and facilitates the process of making decisions a couple makes about the kind of family you want to conform and family life project they plan to build. Service delivery has been described in scientific literature as little inclusive for men, despite the influence they have on the health of their sexual partners and their own sexual health. To determine through the perceptions 
of providers of family planning services if the care provided meets the needs of men. The research is a descriptive study of cross section, made in the Polyclinic "Antonio Maceo". In-depth interview and observation: two techniques were applied. The practice of the providers is little inclusive of the needs of male attention, this can not be interpreted as a lack of intent to those professional about it, but as an expression and reinforcement do more based on social experience, with cultural base which in most contemporary scientific and cultural approaches, gender equality and determination in health. The service provided by health personnel of Family Planning Consultation does not meet care needs related to their sexual and reproductive health of men, but to the attention of those identified by and for women.

Keywords: Family planning. Mem. Care needs.

\section{INTRODUCCIÓN}

La salud sexual y reproductiva es una temática que se ha abordado en las últimas décadas relacionada con los estudios de género. Específicamente en la Conferencia Internacional sobre Población y Desarrollo, se promovió la equidad de género en todas las esferas de la vida, pero aún se está pasando por alto el papel influyente que los hombres desempeñan en la SSR de sus familias y parejas sexuales. (1)

En el caso de los hombres se ha encontrado que su participación en los procesos de la salud reproductiva es pobre, lo cual se atribuye por una parte a que los servicios de Planificación Familiar (PF) han centrado su atención en la mujer, y por otra, a la poca disponibilidad y variedad de métodos anticonceptivos masculinos eficaces. (2)

Este es el contexto en el que se inscribe el escaso número de estudios de SSR vinculados a la problemática masculina. Las investigaciones realizadas han estado abocadas al género, desde una perspectiva teórica y general. La literatura ha sido determinante al analizar el impacto que origina la cultura falocéntrica (idealización del miembro masculino como un ícono de poder y superioridad, esas características están asociadas a su carga simbólica) en la construcción de la identidad masculina latinoamericana. (3)

La relación entre el hombre y su pene va más allá de meras cuestiones sexuales o biológicas. La genitalización de la sexualidad masculina fija comportamientos que hacen de la actividad sexual, el medio necesario para demostrar la asunción del modelo hegemónico de la masculinidad como identidad adoptada. (3)

Cuestiones como la virilidad, la potencia sexual, o el tamaño de los genitales se consideran características propias de lo masculino y al mismo tiempo otorgan poder en las relaciones de pareja, de ahí la importancia que las percepciones masculinas sean consideradas para el diseño de los servicios de SSR; pues es precisamente el varón un componente central en el proceso de toma de decisiones en las relaciones sexuales, lo que está muy interrelacionado con la adopción de métodos anticonceptivos y con la prevención de infecciones de transmisión sexual (ITS) y del VIH. (2) (4)

Esta tendencia a estudiar la sexualidad masculina como forma de identificación, condujo al interés científico hacia temas como la fecundidad, planificación familiar y paternidad, tratados desde la perspectiva de género. (5)

La premura de la incorporación de los hombres a la planificación familiar, también se justifica por la influencia de ellos en la salud sexual y 
reproductiva de la mujer y por los graves daños que ocasiona la reproducción de comportamientos propios de la cultura patriarcal. (6)

Los hombres desempeñan un papel importante en el apoyo y desarrollo de las necesidades de salud de la pareja. Por tanto, si un objetivo agregado de la planificación familiar y de los programas de salud reproductiva es nutrir un proceso de toma de decisión que involucre a ambos miembros de la pareja y respete su interés, claramente estos programas deben estar informados con mediciones más completas y exactas de las visiones del hombre (como las de la mujer). (6)

Uno de los servicios de salud encargados del cuidado de la SSR se denomina Consulta de Planificación Familiar (CPF), ese espacio tiene como objetivo lograr una actuación consciente y responsable de la pareja en el derecho a decidir el número de hijos que se desea tener; además de propiciar la salud materna infantil y la paternidad comprometida. En general, brinda la orientación oportuna y necesaria para la conformación responsable de la unidad básica de toda la estructura social. (7)

Son disímiles los beneficios de ese servicio, aunque estudios interesados por el tema género, evidencian una marcada tendencia hacia la participación casi exclusiva de las mujeres, aun cuando se reconoce la importancia de la participación de los hombres en la planificación familiar (PF) y en la disminución del riesgo reproductivo. (7)

La mayoría de los estudios sobre planificación familiar y participación de los hombres en estos procesos, advierten que las necesidades de información y consejería de los hombres no se suelen tomar en cuenta en la provisión de estos servicios y generalmente son dirigidos a las mujeres, las que acuden en mayor medida. También se han estudiado a los hombres desde una perspectiva de déficit en cuanto a la participación en la atención a la salud sexual, en lugar de aproximarse a la identificación y atención pertinente a las necesidades masculinas sentidas. (6)

Los estudios también han hecho énfasis en la escasez de servicios y programas orientados a las expectativas de los hombres, a la baja participación de los ellos en los mismos, la poca capacitación como usuarios, y a la carencia de personal idóneo para brindar servicios con sensibilidad de género. También han estado encaminados a describir los servicios existentes en busca de posibles inequidades y exponer los factores causantes de las mismas; generalmente desde la perspectiva del prestador de servicio y no de sus usuarios. (8)

La inclusión de los hombres en los procesos de planificación familiar debe partir desde la propia dinámica de la consulta. Las y los prestadores de salud también pueden también estar permeados de las mismas construcciones y representaciones sociales sobre la sexualidad, reproducción, masculinidad, feminidad que las personas que asisten a los espacios donde brindan sus servicios. Identificar las opiniones, criterios de las o los prestadores de salud representaría un paso de avance hacia el aumento de la participación consiente de los hombres en la PF. Determinar a través de las percepciones de los/las prestadores/ as del servicio de planificación familiar si la atención que brindan satisface las necesidades de los hombres es el objetivo del presente artículo.

Los resultados obtenidos serán de utilidad para la toma de decisiones a favor de crear o transformar servicios de salud sexual y reproductiva en repuesta a las necesidades propias de varones y mujeres, posibilitando contar con un espacio verdaderamente inclusivo de las identidades genéricas. 


\section{MÉTODO}

La investigación consistió en un estudio de carácter descriptivo de corte transversal, realizado en el municipio Cerro, ubicado en la provincia $\mathrm{La}$ Habana, durante el período comprendido entre enero 2014 y enero 2015.

Se seleccionó el Policlínico Docente "Antonio Maceo" ubicado en el Consejo Popular Antonio Maceo pues entre los servicios que brinda se encuentra la Consulta de Planificación Familiar. La muestra se eligió a partir de un muestreo no probabilístico "intencional por criterios" de tipo exhaustivo.

El grupo estudiado lo conformaban profesionales de la salud que cumplieron el siguiente criterio de inclusión:

- Que estuvieran vinculados a los servicios de planificación familiar del policlínico Antonio Maceo

Este grupo quedó constituido por una médica y una enfermera

Se emplearon dos técnicas para recabar los datos: entrevista en profundidad semi-estructurada y observación. La aplicación en conjunto de estas técnicas facilitó la detección de la congruencia o discrepancia entre los discursos, las percepciones y los comportamientos. Permitió evidenciar las diferencias entre aquello que se dice y lo que realmente se hace.

Se elaboró una entrevista semi-estructurada y fue validada, en cuanto a comprensión y factibilidad, mediante un estudio piloto realizado con la médica y enfermera de un consultorio médico ubicado en el área de salud estudiada.

La observación se realizó como complemento a la entrevista aplicada.

En la investigación social, esa técnica deviene en un método de recopilación de información social primaria acerca del objeto estudiado mediante la directa percepción y registro de todos los factores significativos concernientes al propósito de la investigación. La literatura científica describe que la observación raras veces se usa como método fundamental de recopilación mas se utiliza junto a otros métodos y sirve a objetivos específicos. (9)

La técnica se aplicó al espacio habilitado para desarrollar la CPF, justamente en el momento en el que se realiza la entrevista. El instrumento que guió la observación fue estructurado.

La observación realizada fue ajena, abierta y estandarizada. (9)

Ajena: la investigadora es quien realiza la observación

Abierta: la investigadora se identifica como tal, las personas que observa conocen que su conducta está siendo observada.

Estandarizada: recogida de datos sobre la base de una guía preestablecida pero no rígida

El constructo emic que emanó del trabajo de campo fue propiciado por la autora a partir de su interacción con las actoras sociales durante el proceso investigativo. Por otra parte, la investigación aportó un producto comprensivo que no es la realidad concreta, sino un constructo descubierto, elaborado bajo el enfoque etic o constitutivo a la investigadora, según su formación académica y humana. Sobre ambos constructos se desarrolló la exposición e interpretación de los resultados. (10) 


\section{ANÁLISIS Y DISCUSIÓN DE LOS RESULTADOS}

El Policlínico Docente "Antonio Maceo" ubicado en el municipio Cerro de la capital cubana atiende un área de salud donde existen 30 consultorios médicos con 9632 familias. (11) Dentro de los servicios allí brindados, la Consulta de Planificación Familiar y Riesgo Reproductivo se encarga de la orientación familiar en el área de la sexualidad de la mujer, el hombre y la pareja. Guía a las personas sobre cómo lograr una actuación consciente y responsable en el derecho de decidir el número de hijos que se desea tener, además de propiciar la salud materno infantil y la paternidad responsable. Ese servicio es brindado por dos especialistas entrenadas para prestar el servicio sobre la base del trabajo multidisciplinario e integral, acción que se garantiza mediante interconsultas con otras especialidades.

Las percepciones de las prestadoras de salud que se identificaron, tienen su base en los datos emanados a partir de la aplicación de las técnicas descritas, pero obedecen a la construcción etic de sus proposiciones de acuerdo con la descripción etic de cada una de las categorías de análisis. (Tabla1).

Tabla 1 - Descripción etic para las categorías de análisis emanadas de los grupos estudiados

\begin{tabular}{|c|c|}
\hline CATEGORÍAS DE ANÁLISIS & DESCRIPCIÓN ETIC \\
\hline $\begin{array}{c}\text { Atención que se presta en planifica- } \\
\text { ción familiar }\end{array}$ & $\begin{array}{c}\text { Objetivos y utilidad de las acciones que conciben y } \\
\text { ejecutan el servicio de PF }\end{array}$ \\
\hline Población beneficiaria & $\begin{array}{c}\text { Personas o grupos a los que se dirige el servicio, } \\
\text { a quienes se enfocan las acciones planteadas en el } \\
\text { programa rector del servicio, según características, } \\
\text { identidades de género }\end{array}$ \\
\hline $\begin{array}{c}\text { Atención a las desigualdades entre } \\
\text { hombres y mujeres }\end{array}$ & $\begin{array}{c}\text { Características en los procesos de atención a las desi- } \\
\text { gualdades de género vinculadas a las acciones que se } \\
\text { plantean para logro de equidad de género en PF }\end{array}$ \\
\hline $\begin{array}{c}\text { Participación de los hombres y } \\
\text { acciones para su fortalecimiento }\end{array}$ & $\begin{array}{c}\text { Concepciones, características y prácticas asociadas } \\
\text { a la participación de los hombres en el servicio de } \\
\text { participación familiar. }\end{array}$ \\
\hline $\begin{array}{c}\text { Necesidades identificadas para } \\
\text { la atención de los hombres y su } \\
\text { satisfacción }\end{array}$ & $\begin{array}{c}\text { Concepciones y prácticas para satisfacer necesidades } \\
\text { de atención de los hombres }\end{array}$ \\
\hline
\end{tabular}

\section{ATENCIÓN QUE SE PRESTA EN PLANIFICACIÓN FAMILIAR}

Durante la conversación con las prestadoras del servicio estudiado, sobre los objetivos y servicios de la consulta, las entrevistadas nunca hicieron alusión al tema hombre, ni siquiera hablaron de los beneficios a la pareja conformada por mujer y hombre. Pareciera que entendieran a la PF como un mecanismo para el control de la fecundidad y si intentamos definir, a partir de sus propias percepciones, el encargo de esa consulta, resultaría correcto inferir que brinda la posibilitad de lograr embarazos saludables en mujeres con riesgo reproductivo presente o no.

Según la doctora y enfermera de este servicio, el propósito final de la atención que se brinda, es el logro de un embarazo planificado con feliz término para la madre y el producto de la concepción.

“...es un servicio multidisciplinario, dirigido a identificar a mujeres en riesgo reproductivo con el objetivo de planificar el momento adecuado para la fecundación y lograr un embarazo deseado. Los servicios brindados también están dirigidos a atenuar y modificar factores que 
provoquen riesgos a la salud reproductiva de esas mujeres." (Doctora de la consulta de planificación familiar)

“...la madre pueda llegar a un embarazo bien planificado, con un buen control y que tenga un resultado de la concepción con buenos parámetros (a término, con buen peso, saludable)." (Doctora de la consulta de planificación familiar)

Entre los servicios de la consulta se encuentran, según sus prestadoras, todas las especialidades que tributen en alguna medida a la disminución y control del riesgo reproductivo preconcepcional de la paciente. Nuevamente, desde su discurso, se relaciona el encargo de la consulta con la atención a la mujer.

“...en la consulta decidimos qué pacientes son las que tienen determinados factores de riesgo. Por ejemplo, si tienen enfermedades crónicas, se interconsulta con las especialidades que van a resolver el factor a modificar." (Doctora de la consulta de planificación familiar)

La poca comprensión de las prestadoras, sobre los temas que atañen a las masculinidades o sobre género en general, puede limitar brindar un servicio de calidad para hombres y mujeres con equidad, pues en el proceso no se identifican sus necesidades y se dirige la atención a satisfacer las de las mujeres. Este es un punto coincidente con investigaciones científicas realizadas en la región. (8)

Los y las profesionales de la salud en Cuba están dotados de conocimientos y mecanismos para enfrentar y darle solución a los problemas de salud de las poblaciones desde un enfoque bio-psico-social. (12) Esta aseveración apunta a que la preparación formal que han recibido estas profesionales, permite detectar en el proceso de atención, aspectos sociales, culturales y psicológicos que participan en la determinación de la salud de los individuos, la familia y las comunidades, que en los procesos de socialización se suelen naturalizar y pueden hacer dirigir la atención hacia un sexo en específico. Sin embargo, no se debe obviar que esas personas están moldeadas por los mismos patrones sociales que sus pacientes, por ello no logran identificar, en la práctica cotidiana, la influencia de factores socioculturales interviniendo en la asistencia masculina. La naturalización de patrones y conductas, conduce la percepción de la realidad y la actuación acorde a esta.

\section{POBLACIÓN BENEFICIARIA}

Las profesionales entrevistadas conciben como población diana del servicio, a las mujeres heterosexuales; para que emergiera en el discurso la pareja heterosexual como población beneficiaria, la investigadora tuvo que indagar explícitamente sobre esta.

“...es un servicio al que debe asistir la pareja, pero no es la generalidad. Son pocas las parejas que vienen juntas. No ocurre lo mismo con el servicio de infertilidad." (Enfermera de la consulta de planificación familiar)

Independientemente que en objetivo de la consulta se declare a la pareja como beneficiaria, las definiciones del servicio brindadas por las prestadoras entrevistadas reduce el encargo de la consulta a la atención de la mujer. La dificultad de las profesionales para referirse, explicar o ejemplificar con acciones, la atención a los hombres como parte de la población diana del servicio puede deberse, a la naturalización de los constructos que guían la atención 
a determinados fenómenos, anclados en las representaciones socioculturales y en la cultura médica (entendida como representaciones socioculturales en salud que expresan a la par de lo normado o científicamente demostrado). (13)

Durante la realización de la entrevista, se observó la existencia de dos sillas en el local donde se realiza la consulta. Una de ellas corresponde a la doctora y la otra es destinada a la/el paciente, la enfermera permaneció de pie. Entonces, un supuesto obvio es que si el servicio está destinado a la atención de la pareja, debería garantizar comodidad para ambos miembros de esta.

De forma similar ocurre con la distribución, mobiliario y características del local donde se lleva a cabo la consulta, a través de lo que se infiere que las atenciones que brinda el servicio se dirigen a la salud de las mujeres: se trata de dos cubículos destinados a la atención, uno para las consejerías u orientaciones a brindar por la doctora y el otro está preparado para la atención ginecobstétrica de la mujer (según explicara la doctora del servicio). No cuenta con algún armario o estante donde estén visibles anticonceptivos masculinos, la decoración está en función de la promoción de la salud de la madre y su descendencia, y ninguno de los afiches hace alusión a la participación masculina en PF., A partir de la descripción de las características del espacio físico de la consulta, pareciera que se confirmara la intención de atender las necesidades de ellas en lugar de satisfacer las de la pareja en su conjunto.

Si bien la praxis institucional expresada o el diseño físico del servicio de PF estudiado, es carente de la suficiente perspectiva de género y poco inclusiva de las necesidades de atención masculina, esto no puede interpretarse como una falta de intención al respecto, sino como expresión y reforzamiento de un hacer basado más en la experiencia social de gestores de salud, con base cultural, que en los enfoques científicos y socioculturales más contemporáneos, sobre equidad de género y su determinación en la salud, especialmente la salud sexual y reproductiva de las poblaciones.

\section{ATENCIÓN A LAS DESIGUALDADES ENTRE HOMBRES Y MUJERES}

La igualdad de hombres y mujeres en la atención en PF es una forma de garantizar los derechos reproductivos de todo habitante. Hoy en día, al mismo tiempo que se sostienen discursos sobre la igualdad, han entrado en escena una valoración muy clara de las diferencias y la diversidad, complejizando el escenario de una manera muy interesante, ya que no se trata de que todos seamos iguales en un sentido estricto (idénticos), sino que exista un proceso de conocimiento y aceptación de las diferencias sin discriminación. (14)

La equidad se hace posible cuando el trato que se le da a las personas está basado en la consideración justa de las necesidades e intereses impuestos por la diferencia, el trato diferenciado es lo que hace posible la equidad. (15)

La falta de inclusión o la presencia de barreras para lograr la participación de los hombres en la CPF se evidencian en los documentos utilizados para evaluar los resultados del servicio. De acuerdo a la observación realizada, las profesionales entrevistadas usan documentos donde solo se recoge información sobre la mujer (edad, paridad, enfermedad que padece la mujer y método anticonceptivo seleccionado)

Esos indicadores deberían revisarse en función de ser adecuados al abordaje médico social que se precisa, inclusivo de la categoría género; a los 
problemas relativos a la salud sexual de las personas sumados a los vinculados a la reproducción biológica, a la que le tributan sin lugar a dudas, y más específicamente los que tienen que ver con la satisfacción de la población en función de sus necesidades.

La no inclusión de los hombres en los registros formales de la consulta los invisibiliza, situación que limita el conocimiento de sus especificidades reproductivas y sexuales, imposibilita documentar transgresiones, sentimientos, estado de ánimo que podrían afectar su salud, la de la pareja y la de la propia familia. (16)

\section{PARTICIPACIÓN DE LOS HOMBRES Y ACCIONES PARA SU FORTALECIMIENTO}

La doctora y enfermera de la consulta admitieron que la participación masculina es fundamental para la dinámica del servicio, pues en la modificación de los posibles factores que representan riesgo a la salud sexual de las parejas es "indispensable la participación de los hombres". (Doctora de la consulta de planificación familiar)

Las entrevistadas comentaron que "la asistencia masculina casi es nula" (enfermera de la consulta de planificación familiar) y ese comportamiento masculino lo explican "por el déficit de educación sexual que origina en la población la idea que la responsabilidad de la maternidad es solo de la mujer”. (Doctora de la consulta de planificación familiar)

Las prestadoras del servicio no identifican entre posibles explicaciones para la conducta de los hombres, la influencia de patrones impuestos por la cultura patriarcal. A pesar de lo planteado, opinan que los hombres consideran más importante su trabajo y no la responsabilidad de asistir a la consulta de $\mathrm{PF}$, pero no identifican esa conducta como resultante de esa división social de funciones. (17) La importancia del trabajo como fuente para obtener los recursos con que sostener la familia es un constructo lógico no solo para hombres sino también para las mujeres, aunque de acuerdo a los roles de género esto tenga una connotación singular para ellos.

“... y ellos les dan más valor a sus responsabilidades laborales debido al desconocimiento que tiene de la importancia de su presencia en las consultas junto a sus parejas." (Doctora de la consulta de planificación familiar)

“...son pocos los que acuden y los que lo hacen no son constantes con su asistencia, pues alegan que están trabajando y eso les imposibilita asistir." (Doctora de la consulta de planificación familiar)

La división del trabajo por sexo es reconocida en la literatura científica, como el eje de las diferencias sociales entre las mujeres y los hombres. Las actividades remuneradas son descritas como realizadas predominantemente por los hombres, mientras las actividades domésticas y de cuidado de los miembros de la familia dentro del hogar son desempeñadas, sin remuneración, primariamente por las mujeres. Las mujeres, producto de transformaciones sociales y la evolución del pensamiento de las personas, están ingresando crecientemente al mercado de trabajo remunerado, aunque las responsabilidades domésticas y familiares continúan recayendo básicamente bajo su dominio. (18) 
Independientemente de lo infrecuente que resulta la asistencia masculina a la consulta, las entrevistadas comentaron sobre la asistencia de algunos hombres durante el año 2014, pero los datos que prueban su presencia no están disponibles en los documentos oficiales del servicio. Las hojas de cargo solo contienen información de las mujeres, una vez más la omisión deviene en invisibilización.

\section{NECESIDADES MASCULINAS IDENTIFICADAS}

Las mujeres y los hombres comparten un conjunto de necesidades comunes a ambos sexos, pero también, tienen necesidades específicas que se derivan de las características biológicas propias de cada sexo, de los distintos roles que desempeñan socialmente, y de la posición diferencial de poder que ocupan dentro de la familia y la sociedad. (19)

En relación a las necesidades de atención en SSR, las mujeres tienen necesidades específicas que han sido históricamente desatendidas por las sociedades, y que esta desatención es tanto una causa como un resultado de formas específicas de discriminación dirigidas contra ellas. Tal énfasis en las mujeres no debe desconocer, el papel imprescindible que juegan los hombres en la transformación de las relaciones de género. (18)

La identificación y satisfacción de las necesidades de atención de los hombres fue también una problemática abordada en la conversación efectuada. Se descubrió que las entrevistadas, entendían como satisfacción de las necesidades, fundamentalmente la orientación efectiva sobre métodos anticonceptivos (a las mujeres).

Durante la entrevista se pudo percibir la dificultad del personal médico para responder preguntas relativas a la atención de los hombres y continuaron reafirmando que la escasa asistencia se debe precisamente al déficit de educación en sexualidad de los hombres.

Todo ello vuelve a transitar por las representaciones y concepciones de estas prestadoras, ancladas en el propio contexto patriarcal en el que viven y se han formado, que se ha reforzado a partir de la experiencia médico social de estas. Esa influencia no les permite percatarse, por ejemplo, que la simple acción de no registrar la visita de un hombre solo o en compañía de su compañera sentimental, les impide satisfacer las necesidades de atención de esa persona y por ende atenta contra la calidad del propio servicio que brindan.

Como se menciona en la introducción, la bibliografía sobre la relación SSR y hombres resulta escasa, situación que repercute directamente en la insuficiencia de acciones concretas para la inclusión de los hombres a esos procesos. Los resultados obtenidos en la presente investigación reflejan coincidencias con otros estudios realizados. Las percepciones de las prestadoras estudiadas son resultantes de su representación de de la masculinidad basada en el modelo hegemónico, es decir, están vinculadas con la manera en que ellas entienden la identidad masculina con respecto a conceptos como sexo, sexualidad, anticoncepción y planificación familiar, independientemente de su formación como profesionales de la salud. (6)

Interpretar estas normas sociales como naturales aleja a prestadores y prestadores de la posibilidad de brindar servicios verdaderamente equitativos. La reproducción de patrones patriarcales mezclado con la imposibilidad de 
detectarlos en la cotidianidad limita el acceso de los hombres a los servicios y por ende, resta calidad a la atención pues no se logra brindar a toda persona sin distinción y por demás no completa el enfoque a la pareja. (17)

Queda camino por recorrer, los hombres y las mujeres no son individuos similares, por tanto esas diferencias biológicas, sexuales, culturales y sociales tienen implicaciones respecto a cómo y cuáles son sus problemas de salud; por tanto, también en cuanto a sus necesidades de intervención sanitaria y social. (3)

\section{CONCLUSIONES}

El servicio brindado por el personal de salud de la Consulta de Planificación Familiar no satisface las necesidades de atención relativas a su salud sexual y reproductiva de los hombres, sino a la atención de las identificadas por y para mujeres.

La atención brindada en la Consulta de Planificación Familiar no responde al encargo social para el cual está diseñada, la atención a las mujeres, vista desde la praxis de las prestadoras hasta el diseño del espacio físico del servicio, no responde a garantizar la satisfacción de las necesidades de atención de hombres y mujeres por igual.

\section{BIBLIOGRAFÍA}

CENTRO DE PRENSA. Planificación familiar. Nota descriptiva [internet] 2013 [citado octubre 2014]; (351) Disponible en: <http://www.who.int/ mediacentre/factsheets/fs351/es/>. Último acesso em: 03/12/2016.

DIAZ Z. Representaciones socioculturales de la infertilidad y su atención en los servicios de salud. Tesis para optar grado doctor en ciencias, 2012.

GABA M. La organización generizadas. La perspectiva de género en acción en el mundo de las organizaciones. En: Tájer D. Género y Salud. Las políticas en acción. Buenos Aires, Argentina: Editorial Lugar, 2012.

GARCÍA P. Políticas de igualdad, equidad y gender maistreaming. ¿De qué estamos hablando? En: PNUD. Gestión del conocimiento para la equidad de género en Latinoamérica y El Caribe. [internet] 2008 [citado enero 2015] Disponible en: <http://www.americalatinagenera.org/es/documentos/ doc_732_Politicasdeigualdad23junio08.pdf $>$. Último acesso em: 03/12/2016. GONZÁLEZ JC. Masculinidades, generando el debate. En: Macho, varón, masculino. Estudios de masculinidades en Cuba. Editorial de la mujer. p. 9-18, 2010.

GÜEZMES A. Reforma del sector salud y derechos sexuales y reproductivos. Washington, D.C: Editorial OPS/PALTEX, 2004.

HERRERA G., RODRÍGUEZ L. Masculinidad y equidad de género: desafíos para el campo del desarrollo y la salud sexual y reproductiva. En: Masculinidades en Ecuador. Editorial Flacso/UNFPA. 19 de abr., 2010.

HERNÁNDEZ-SAMPIERI R, FERNÁNDEZ-COLLADO C, BAPTISTA P. Metodología de la Investigación. Editorial McGraw-Hill Interamericana: México DF, 2006.

IBARRA F y coautores. Metodología de la investigación social. La Habana: Editorial Félix Varela, 2010. 
MENDOZA D. Planificación familiar: logros en la última década y retos futuros. [internet] 2006 [citado noviembre 2014]. Disponible en:

$<$ http://www.portal.conapo.gob.mx/publicaciones/sdm/sdm2006/sdm06_04. pdf $>$. Último acesso em: 03/12/2016.

OFICINA DE ESTADÍSTICA DEL POLICLÍNICO DOCENTE “ANTONIO MACEO”. Dispensarización año 2014.

OPS/OMS. Guía para el análisis y el monitoreo de la equidad de género en las políticas de salud. [internet] 2008 [citado noviembre 2013]; Disponible en: $<$ http://publications.paho.org/product.php?productid=946> . Último acesso em: 03/12/2016.

ORGANIZACIÓN MUNDIAL DE LA SALUD. Conferencia Internacional de Población y Desarrollo. El Cairo: OMS; 1994.

RODRÍGUEZ V., CASTAÑEDA I.E, RODRÍGUEZ A., DÍAZ Z, LOZANO

A. Necesidad del abordaje de los estudios de la salud sexual y reproductiva en el hombre. Revista Cubana de Salud Pública v. 39(1), p. 65-69, 2013.

TÁJER D. GÉNERO Y SALUD. Las políticas en acción. Editorial Lugar. Argentina, 2012.

. Plan Estratégico de Transversalización. Perspectiva de Género en Salud 2014-2018 en el marco del Plan de Salud de Centroamérica y República Dominicana. [internet] 2014 [citado noviembre 2014] Disponible en: $<$ http://www.cide.edu/docs/buenas-practicas/equidad-genero/PPSalud.pdf $>$. Último acesso em: 03/12/2016.

TORRES W. Comportamiento sexual y reproductivo en hombres. Un estudio comparativo a partir de territorio de residencia. En: Género salud y sexualidad. Cedem, 2013.

VELA J. Modificaciones de las asignaturas de Salud Publica en el plan de estudio perfeccionado de la carrera de medicina. 1985-2010. Trabajo para optar por el título de máster en educación médica. La Habana, 2011. 
